(c) American Dairy Science Association, 2006.

\title{
Effects of a Lactobacillus casei Synbiotic on Serum Lipoprotein, Intestinal Microflora, and Organic Acids in Rats
}

\author{
M. T. Liong and N. P. Shah ${ }^{1}$ \\ School of Molecular Sciences, Victoria University, Werribee Campus, PO Box 14428, Melbourne, 8001, Australia
}

\begin{abstract}
The main aim of this study was to evaluate the effectiveness of 3 synbiotic diets: 1) containing Lactobacillus casei ASCC 292 and fructooligosaccharides (LF diet); 2) containing L. casei ASCC 292 and maltodextrin (LM diet); and 3) containing $L$. casei ASCC 292, fructooligosaccharide, and maltodextrin (LFM diet) to reduce serum cholesterol in male Wistar rats. The effect of the synbiotic diets on intestinal microflora, concentration of organic acids, and the possibility of translocation of lactobacilli were also investigated. The LFM diet lowered serum total cholesterol and triglyceride levels, whereas the LM diet increased serum high-density lipoprotein cholesterol level. However, synbiotic diets did not contribute to a change in low-density lipoprotein cholesterol level compared with the control diet. There was a decrease in the population of staphylococci, bacteroides, Escherichia coli, and total coliforms in most bowel regions with the LFM diet compared with the control (which did not contain any synbiotic). In general, the LFM diet contributed to a higher concentration of lactic acid that may have contributed to the decrease in the population of pathogenic microorganisms compared with the control. Fructooligosaccharide was the preferred substrate for production of acetic acid. Results from this study showed that the synbiotic diet that contained $L$. casei ASCC 292, fructooligosaccharide, and maltodextrin beneficially altered cholesterol levels and produced a healthier bowel microbial population without translocation of lactobacilli to other organs.
\end{abstract}

Key words: fructooligosaccharide, maltodextrin, lactobacilli, cholesterol

\section{INTRODUCTION}

Epidemiological studies have shown that higher than normal serum total cholesterol or low-density lipoprotein (LDL) cholesterol increased the risk of coronary heart disease (Usman and Hosono, 2000). Lactic acid bacteria,

Received November 3, 2005.

Accepted December 1, 2005.

${ }^{1}$ Corresponding author: nagendra.shah@vu.edu.au especially lactobacilli, are probiotics that are considered potentially useful in their role to reduce serum cholesterol. Grunewald (1982) found that rats fed milk fermented with Lactobacillus acidophilus for 4 wk showed lowered serum cholesterol levels compared with the control group that was fed only unfermented milk. Several mechanisms are believed to be involved in the reduction of serum cholesterol. Our previous studies reported that lactobacilli are capable of removing cholesterol in vitro via various mechanisms; namely assimilation, binding to surface of cells, incorporation into cellular membrane, and coprecipitation with deconjugated bile (Liong and Shah, 2005a,b). Probiotic numbers are enhanced by prebiotics, which are defined as "nondigestible food ingredients that beneficially affect the host by selectively stimulating the growth and activity of one or a limited number of bacterial species already resident in the colon, and thus improving host health" (Gibson and Roberfroid, 1995). Several classes of resistant starch, fiber, oligosaccharides, and sugar alcohols are classified as prebiotics. Fiber sources such as oat bran reportedly lowered plasma cholesterol in rats through enhancing steroid excretion that was accelerated by increased production of propionate (Chen et al., 1984).

The use of both probiotics and prebiotics (known as synbiotics) as a natural means to counter increased cholesterol levels has generated much interest recently. Using in vitro experiments, we have previously screened and developed a synbiotic product comprising a Lactobacillus casei strain and the prebiotics fructooligosaccharide (FOS) and maltodextrin that specifically targeted removal of cholesterol in laboratory media (Liong and Shah, 2005c). Further studies are needed to evaluate its effect in in vivo models. Although reports have cited positive effects on the use of probiotics, prebiotics, or synbiotics in reducing serum cholesterol levels, they have also noted controversial results. Grunewald and Mitchell (1983) previously reported that milk fermented by $L$. acidophilus exerted no hypocholesterolemic effect on rats, whereas Thompson et al. (1982) reported that acidophilus milk did not reduce serum cholesterol levels in humans. Resistant starch showed cholesterol-lowering properties in rats but did not affect plasma cholesterol in humans (Jenkins et al., 1987). 
Probiotic bacteria such as Lactobacillus are generally regarded as safe (GRAS) for consumption. Until now, reports of harmful effects of these microbes toward a host are rare and their safety has not been questioned. However, there have been reports of probiotic strains such as L. casei, Lactococcus lactis, and Lactobacillus plantarum being isolated from bacterial enterocarditis and Bifidobacterium adolescentis being isolated from blood stream infections (Gasser, 1994). These incidences indicate that these bacteria are capable of translocating from the intestine to other organs. Although it is difficult to induce such translocation in healthy animals, it could occur if the gut environment was altered by intestinal mucosa injury, immunodeficiency in the host, or abnormal intestinal bacterial flora (Ishibashi and Yamazaki, 2001). Although the $\mathrm{pH}$ of the stomach may reach as low as 1.5 , the $\mathrm{pH}$ of the lower intestine is near neutral (Liong and Shah, 2005a). High concentrations of organic acids, arising from rapid fermentation of prebiotics by probiotics that inhibit the colonization of acid-sensitive pathogens, could also induce injury to the intestinal mucosa and hence, impair its barrier function (Argenzio and Meuten, 1991; Remesy et al., 1993). Thus, it is of utmost importance to ensure that the synbiotics developed are safe and have no indication of harmful translocation.

Other than exerting cholesterol-lowering effects, lactobacilli and prebiotics are associated with the alteration of the intestinal flora population and have been used to suppress growth of pathogens through competitive inhibition, production of short-chain fatty acids (SCFA), and antagonistic activity against pathogens (Buddington et al., 2002; Isolauri et al., 2002). Our previous study (Liong and Shah, 2005c) reported that $L$. casei ASCC 292 was not only capable of removing cholesterol in vitro but also of producing SCFA in the presence of FOS and maltodextrin. Thus, the aim of this study was to evaluate the effectiveness of such synbiotics on reducing serum cholesterol using a rat model. The effect of the synbiotics on intestinal microflora, concentration of organic acids, and the capability to translocate was also investigated.

\section{MATERIALS AND METHODS}

\section{Source of Culture and Prebiotics}

Lactobacillus casei ASCC 292, a human-derived strain, was obtained from the Australian Starter Culture Collection Center (ASCC, Werribee, Australia). The stock culture was stored in $40 \%$ (vol/vol) glycerol at $-80^{\circ} \mathrm{C}$. The organism was subcultured 3 times before use in sterile de Man, Rogosa, Sharpe broth using $1 \%$ inoculum and $20 \mathrm{~h}$ of incubation at $37^{\circ} \mathrm{C}$, and was stored at $4^{\circ} \mathrm{C}$ between transfers. Freeze-dried culture in a powder form (approximately $9.0 \log _{10} \mathrm{cfu} / \mathrm{g}$ ) was used in this
Table 1. Composition of the high-cholesterol diet (SF00-245) that contained $16 \%$ fat, $1 \%$ cholesterol, and $0.5 \%$ cholate (Specialty Feeds, Glen Forrest, Australia)

\begin{tabular}{lr}
\hline Ingredients, g/kg & \\
\hline Casein & 200 \\
Methionine & 3 \\
Sucrose & 520 \\
Cellulose & 51 \\
Canola oil & 10 \\
Cocoa butter & 150 \\
Calcium carbonate & 13.1 \\
Sodium chloride & 2.6 \\
Potassium citrate & 2.5 \\
Potassium dihydrogen phosphate & 6.9 \\
Potassium sulphate & 1.6 \\
AIN93G trace minerals & 1.4 \\
Choline chloride $(65 \%)$ & 10 \\
Sodium cholate & 5 \\
Cholesterol (USP) & 10 \\
AIN93G vitamins & 10 \\
$\alpha$-Tocopherol acetate $(50 \%$ active) & 2.6 \\
\hline
\end{tabular}

study. Thus, the cell pellet obtained from the fermentation broth was suspended in $0.1 M$ phosphate buffer $(\mathrm{pH}$ 6.8) containing $2.0 \%$ (wt/vol) of food-grade cryoprotectant Unipectin RS 150 (Savannah Bio Systems, Balwyn East, Australia). The mixture was frozen at $-20^{\circ} \mathrm{C}$ before freeze-drying (Dynavac FD300 freeze-dryer; Airvac Engineering Pty. Ltd., Rowville, Australia) at $-20^{\circ} \mathrm{C}$ and $-100 \mathrm{kPa}$. Two commercially available prebiotics were used, including FOS (Raftilose P95, Orafti Pty. Ltd., Tienen, Belgium) and maltodextrin (Grain Processing Corp., Muscatine, IA). The FOS used was extracted from chicory with a purity of $95 \%$; the remaining $5 \%$ contained glucose, fructose, and sucrose. The degree of polymerization of oligofructose ranged from 2 to 7 , with an average degree of polymerization of 4 . The maltodextrin used was Maltrin M100, a glucose polymer with dextrose equivalent ranging from 9 to 12 and an average degree of polymerization of 11 .

\section{Rats and Diets}

Conventional male Wistar rats $(\mathrm{n}=24$; Monash University Animal Services, Clayton, Australia) at $8 \mathrm{wk}$ of age were used. The rats were housed and bred as approved by the Animal Ethics Committee of Victoria University (Werribee, Australia). Upon arrival, the animals were kept on rodent chow for a week. After this washout period, rats were divided into 4 groups of 6 rats in each group $(n=6)$. Rats were kept separately in metal cages in a room with controlled temperature $\left(20\right.$ to $22^{\circ} \mathrm{C}$ ) and humidity (50 to 55\%), and maintained in a cycle of light for $12 \mathrm{~h}(0600$ to $1800 \mathrm{~h})$ and dark for $12 \mathrm{~h}(1800$ to $0600 \mathrm{~h}$ ). The composition of the high-cholesterol diet that contained 16\% (wt/wt) fat, 1\% (wt/wt) cholesterol, and 0.5\% (wt/wt) cholate (SF00-245, Specialty Feeds; Glen Forrest, Australia) is shown in Table 1. Rodent chow 
Table 2. The composition of control and treatment $\operatorname{diets}^{1}$

\begin{tabular}{|c|c|c|c|c|}
\hline \multirow[b]{2}{*}{ Ingredients (g/kg) } & \multicolumn{4}{|c|}{$\operatorname{Diet}^{1}$} \\
\hline & Control & $\mathrm{LF}$ & LM & LFM \\
\hline SF00-245 ${ }^{2}$ & 750.0 & 750.0 & 750.0 & 750.0 \\
\hline Copha $^{3}$ & 50.0 & 50.0 & 50.0 & 50.0 \\
\hline L. casei ASCC 292 & - & 8.5 & 8.5 & 8.5 \\
\hline $\mathrm{FOS}^{4}$ & - & 24.0 & - & 24.0 \\
\hline Maltodextrin ${ }^{4}$ & - & - & 34.0 & 34.0 \\
\hline Rodent chow ${ }^{5}$ & 200.0 & 167.5 & 157.5 & 133.5 \\
\hline
\end{tabular}

${ }^{1}$ Diets: $\mathrm{LF}=$ Lactobacillus casei ASCC 292 and fructooligosaccharides; $\mathrm{LM}=$ L. casei ASCC 292 and maltodextrin; and LFM = L. casei ASCC 292, fructooligosaccharide, and maltodextrin.

${ }^{2}$ High-cholesterol diet (Specialty Feeds, Glen Forrest, Australia).

${ }^{3}$ Copha (Unilever Australasia, Epping, Australia); hardened coconut oil $99 \%$, soybean lecithin $1 \%$.

${ }^{4}$ Fructooligosaccharide (Raftilose P95; Orafti Pty. Ltd., Tienen, Belgium), purity of $95 \%$, average degree of polymerization of 4 ; Maltodextrin (Maltrin M100; Grain Processing Corp., Muscatine, IA), dextrose equivalent of 9 to 12 , average degree of polymerization of 11 .

${ }^{5}$ Rodent chow (Specialty Feeds, Glen Forrest, Australia), total protein $19.6 \%$, total fat $4.6 \%$, crude fiber $4.8 \%$, trace minerals $2.9 \%$, total vitamins $0.3 \%$.

was incorporated into the diet to maintain a similar amount of SF00-245 in all groups that had varying amount of synbiotics. Solidified coconut oil (Copha, Unilever Australasia, Epping, Australia) was added to aid the pelleting process of feed production.

\section{Experimental Design}

All groups were on diets containing $75 \%$ (wt/wt) of the high-cholesterol diet and 5\% (wt/wt) copha (hardened coconut oil 99\%, soybean lecithin 1\%). Group 1 was fed the control diet containing SF00-245, copha, and rodent chow. Group 2 received a cholesterol-enriched diet plus L. casei ASCC 292 and FOS (LF); group 3 received a cholesterol-enriched diet plus L. casei ASCC 292 and maltodextrin (LM); and group 4 was fed the cholesterolenriched diet with L. casei ASCC 292, FOS, and maltodextrin (LFM). The composition of all 4 diets is shown in Table 2. The composition was in accordance with our previous in vitro optimization study; inoculum size of L. acidophilus ATCC 4962, concentrations of FOS, and maltodextrin were the significant factors for optimum removal of cholesterol, and was achieved from the ratio of 1.7:4.8:6.8 (Liong and Shah, 2005c). Rats were allowed to consume their respective diets and water ad libitum for 6 wk. Body weight and feed intake were recorded weekly.

\section{Sampling and Analytical Procedures}

At the end of the 6-wk feeding trial, rats were fasted overnight, and killed by carbon dioxide inhalation. The cecum was tied off immediately after dissection to avoid leakage of cecal contents into the colon due to relaxed gut muscle upon death. Blood samples were collected immediately in sterile tubes by heart puncture, and left to stand for $30 \mathrm{~min}$ at room temperature $\left(\sim 20^{\circ} \mathrm{C}\right)$ to coagulate before being centrifuged for $20 \mathrm{~min}$ at $2,714 \times g$ (Sorvall RT7, Newtown, MA). The serum samples were analyzed for total, high-density lipoprotein (HDL), and LDL cholesterol, and triglycerides using commercial and reagent kits (Thermo Electron Corp., Melbourne, Australia).

Fecal samples were collected weekly in separate sterile tubes for microbial and organic acids analyses. Samples for microbial analyses were placed into anaerobic jars (Becton Dickinson Microbiology Systems, Sparks, MD) with gas-generating kits (Oxoid Ltd., Hampshire, UK) and analyses were carried out within $1 \mathrm{hr}$ of collection. Each sample was homogenized with a stomacher (John Morris Scientific Pty. Ltd., Melbourne, Australia) using sterile peptone and water diluents. Subsequent 10 -fold serial dilutions of each sample were plated in triplicate. All enumeration media were obtained from Amyl Media Pty. Ltd. (Dandenong, Australia). Nutrient agar was used for total aerobes, whereas Wilkins-Chalgren agar was used for total anaerobes (McBain et al., 2003). Eosin methylene blue agar was used for $E$. coli (Swanson et al., 2002), esculin bile salt agar for bacteroides (Tannock et al., 2000), tryptose sulfite cycloserine agar for clostridia (Wise and Siragusa, 2005), mannitol salt agar for staphylococci (Knowles et al., 2005), violet red bile agar for coliform (Cotton and White, 1992), and de Man, Rogosa, Sharpe agar (Merck KGaA, Darmstadt, Germany) for total lactobacilli (Swanson et al., 2002). Plates of total anaerobes, bacteroides, clostridia, and lactobacilli were incubated anaerobically at $37^{\circ} \mathrm{C}$ for 48 $\mathrm{h}$ in anaerobic jars with gas-generating kits. Plates for the enumeration of total aerobes, $E$. coli, staphylococci, and coliforms were incubated at $37^{\circ} \mathrm{C}$ for $48 \mathrm{~h}$ in controlled aerobic incubators (New Brunswick Scientific, Edison, NJ).

Upon dissection, the contents of colon and cecum were collected, stored anaerobically, and analyzed for microbial analyses in a similar manner as fecal samples. The spleen, liver, and kidneys were removed, blotted on a filter paper, and immediately kept in separate sterile tubes. All spleens, livers, and kidneys were analyzed for the presence of lactobacilli to ascertain translocation.

All collected fecal, cecal, and colon samples were stored at $-40^{\circ} \mathrm{C}$ until analyzed for organic acid contents. The concentration of organic acids was determined using HPLC (Varian Australia Pty. Ltd., Mulgrave, Australia). Samples were prepared for HPLC analysis as described previously (Dubey and Mistry, 1996). Acetic, propionic, butyric, formic, and lactic acids were identified using respective standards, and their concentrations deter- 
Table 3. Body weight gain, feed intake, and feed efficiency of rats fed control and synbiotic diets ${ }^{1}$

\begin{tabular}{|c|c|c|c|c|}
\hline \multirow[b]{2}{*}{ Component } & \multicolumn{4}{|c|}{ Treatment $^{2}$} \\
\hline & Control & $\mathrm{LF}$ & LM & LFM \\
\hline BW gain (g/rat per wk) & $34.001 \pm 2.404^{\mathrm{ab}}$ & $33.692 \pm 1.013^{\mathrm{ab}}$ & $39.244 \pm 2.188^{\mathrm{a}}$ & $30.701 \pm 3.127^{\mathrm{b}}$ \\
\hline Feed intake $(\mathrm{g} / \mathrm{wk})$ & $140.532 \pm 4.011^{\mathrm{a}}$ & $136.785 \pm 4.081^{\mathrm{a}}$ & $148.064 \pm 4.071^{\mathrm{a}}$ & $131.066 \pm 4.071^{\mathrm{a}}$ \\
\hline BW:feed intake & $0.245 \pm 0.041^{\mathrm{a}}$ & $0.254 \pm 0.051^{\mathrm{a}}$ & $0.272 \pm 0.050^{\mathrm{a}}$ & $0.239 \pm 0.014^{\mathrm{a}}$ \\
\hline
\end{tabular}

mined. The HPLC system was equipped with a UV/Vis detector (Varian, Walnut Creek, CA) set at $220 \mathrm{~nm}$. An Aminex HPX-87H column (BioRad Laboratories, Richmond, VA) was maintained at $65^{\circ} \mathrm{C}$ and the degassed mobile phase $\left(0.009 \mathrm{M} \mathrm{H}_{2} \mathrm{SO}_{4}\right)$ was used at a flow rate of $0.6 \mathrm{~mL} / \mathrm{min}$.

The moisture in feces and cecal and colon contents was determined as the difference between the wet mass and the dry mass of the samples after drying at $80^{\circ} \mathrm{C}$ (Memmert GmbH Ltd., Schwabach, Germany) until a constant weight was achieved. The $\mathrm{pH}$ of the samples was measured with a $\mathrm{pH}$ meter (Hanna Instruments Pty. Ltd., Kallang Way, Singapore).

\section{Statistical Analyses}

The data analysis was carried out with SPSS Inc. software (version 10.0). One-way ANOVA was used to study a significant difference between means of the dietary groups at a given intestinal site and sampling sites within the same dietary group, with a significance level of $P<0.05$. Tukey's-test was used to perform multiple comparisons between means. All data are presented as mean \pm standard error of means; $n=6$.

\section{RESULTS}

\section{Weight and Feed Intake}

All rats were generally healthy throughout the feeding trial period. Despite differences in weight gain between rats on diet LM and those on diet LFM, the feed intake and the ratio of body weight to feed intake showed no significant difference $(P>0.05)$ between synbiotic treatment groups and the control (Table 3 ).

\section{$\mathrm{pH}$ and Moisture Content of Fecal, Cecal, and Colon Contents}

All groups showed a gradient increase in $\mathrm{pH}$ values from cecal content to fecal samples (Table 4). The $\mathrm{pH}$ of cecal and colon content was not significantly different
$(P>0.05)$ among all treatment groups and control. However, the $\mathrm{pH}$ of the fecal samples of rats fed diet LM was lower compared with that with the control diet.

All rats showed a significant difference $(P<0.05)$ in moisture content from cecal to fecal samples (Table 5). Rats fed the LM and LFM diets had higher moisture content in the fecal samples compared with those on the control diet.

\section{Translocation of Lactobacilli}

Samples from spleen, liver, and kidney of each rat on the control, LF, LM, and LFM diets were plated for the presence of total lactobacilli. No growth of lactobacilli was detected from the samples indicating the absence of translocation (data not shown).

\section{Lipid Profiles}

Serum total cholesterol, triglycerides, HDL, and LDL cholesterol levels of rats fed the control, LF, LM, and LFM diets are shown in Figure 1. Rats fed the LFM diet had significantly $(P<0.05)$ lower total cholesterol and triglyceride levels compared with the control, whereas rats fed the LF or LM diets did not show any difference. The HDL cholesterol level increased in serum of rats that were fed the LM diet compared with the control diet. However, diets containing FOS and a mixture of both FOS and maltodextrin did not increase the serum HDL cholesterol level. Although rats fed the LF and LFM diets showed lower serum LDL cholesterol level, all synbiotic diets had similar effects on LDL cholesterol level compared with the control diet.

\section{Microbial Populations}

Total aerobes ranged from 7.156 to $9.929 \mathrm{cfu} \log _{10} / \mathrm{g}$ of dry weight of cecal, colonic, and fecal samples of rats across all treatment diets (Table 6). A gradient decrease in total aerobes was observed from cecal to fecal samples, with fecal samples from all diets showing lower total 
Table 4. $\mathrm{pH}$ values of contents obtained from the cecum, colon, and feces of rats fed control and synbiotic $\operatorname{diets}^{1}$

\begin{tabular}{lllll}
\hline & \multicolumn{4}{c}{ Treatment $^{2}$} \\
\cline { 2 - 5 } Sample & Control & LF & LM & LFM \\
\hline Cecum & $6.43 \pm 0.06^{\mathrm{b}, \mathrm{A}}$ & $6.28 \pm 0.05^{\mathrm{b}, \mathrm{A}}$ & $6.49 \pm 0.06^{\mathrm{a}, \mathrm{A}}$ & $6.36 \pm 0.04^{\mathrm{b}, \mathrm{A}}$ \\
Colon & $6.79 \pm 0.04^{\mathrm{b}, \mathrm{A}}$ & $6.37 \pm 0.18^{\mathrm{b}, \mathrm{A}}$ & $6.51 \pm 0.02^{\mathrm{a}, \mathrm{A}}$ & $6.64 \pm 0.04^{\mathrm{b}, \mathrm{A}}$ \\
Feces & $7.80 \pm 0.08^{\mathrm{a}, \mathrm{A}}$ & $8.13 \pm 0.13^{\mathrm{a}, \mathrm{A}}$ & $7.04 \pm 0.02^{\mathrm{a}, \mathrm{B}}$ & $7.79 \pm 0.01^{\mathrm{a}, \mathrm{A}}$ \\
\hline
\end{tabular}

${ }^{\mathrm{a}, \mathrm{b}}$ Means in the same column followed by different lowercase letters are significantly different $(P<0.05)$.

${ }^{\mathrm{A}, \mathrm{B}}$ Means in the same row followed by different uppercase letters are significantly different $(P<0.05)$.

${ }^{1}$ Results are expressed as mean \pm standard error of means, $\mathrm{n}=6$.

${ }^{2}$ Treatments: Control $=$ no L. casei ASCC 292, fructooligosaccharide (FOS), or maltodextrin; LF $=0.9 \%$ (wt/wt) L. casei ASCC 292 and 2.5\% (wt/wt) FOS; LM = 0.9\% (wt/wt) L. casei ASCC 292 and 3.3\% (wt/wt) maltodextrin; LFM = 0.9\% (wt/wt) L. casei ASCC 292, 2.5\% (wt/wt) FOS, and 3.3\% (wt/wt) maltodextrin.

aerobe counts compared with the samples from other intestinal sites. Rats supplemented with the LFM diet showed a lower concentration of total aerobes in the colonic samples compared with the control rats. Staphylococci averaged from 6.184 to $9.149 \mathrm{cfu} \log _{10} / \mathrm{g}$ of dry weight of all samples. The LF and LM diets showed a significant decrease in staphylococci after the colon and cecal regions, respectively, whereas the control showed similar staphylococci counts along the intestinal gradients. Rats supplemented with the LFM diet showed a lower concentration of staphylococci in the colonic and fecal samples compared with the control. A gradient decrease of $E$. coli was also observed from cecal samples to fecal samples across all diets. Rats supplemented with the LF diet showed lower counts of $E$. coli in the fecal samples compared with the control rats, whereas the LFM diet lowered total count of $E$. coli in the cecal and colon regions. Total coliforms ranged from 6.757 to 9.215 cfu $\log _{10} / \mathrm{g}$ of dry weight of all samples studied. Fecal samples of all diets showed a lower concentration of coliforms compared with other intestinal sites. Total coliforms were reduced from the upper intestinal sites of rats supplemented with the LFM diet compared with those fed the control diet.

The concentration of total anaerobes of rats fed the control, LF, LM, and LFM diets is shown in Table 7.
Only rats supplemented with the LF and LFM diets showed a significant decrease in total anaerobes after the colonic region, whereas the control rats had similar total anaerobic counts across all intestinal sites. Concentrations of clostridia averaged from 3.618 to $5.101 \mathrm{cfu}$ $\log _{10} / \mathrm{g}$ of dry weight across all samples studied. Most diets showed a consistent population of clostridia along all intestinal sites. However, the LFM diet produced a lower concentration of clostridia in the cecum and colonic regions compared with the control. All diets showed decreasing counts of bacteroides along the intestinal gradients. The LFM diet contributed to a decrease of bacteroides after the cecal region whereas the control diet only reduced the concentration of bacteroides in the lower colonic regions. Most of the synbiotic diets reduced the concentration of bacteroides in all intestinal gradients studied compared with the control. Rats fed with LF and LFM diets maintained a consistent concentration of lactobacilli across all intestinal sites studied, whereas the control diet showed a gradient decrease after the colonic region. Rats fed the LF diet also showed a higher concentration of lactobacilli in the fecal samples compared with the control, whereas the LM diet contributed to a higher count of lactobacilli in most of the intestinal samples compared with the control.

Table 5. Moisture content (\%) of cecal, colon, and fecal content of rats fed control and synbiotic diets ${ }^{1}$

\begin{tabular}{lllll}
\hline & \multicolumn{4}{c}{ Treatment $^{2}$} \\
\cline { 2 - 5 } Sample & Control & LF & LM & LFM \\
\hline Cecum & $77.574 \pm 1.632^{\mathrm{a}, \mathrm{A}}$ & $87.836 \pm 2.144^{\mathrm{a}, \mathrm{A}}$ & $82.184 \pm 3.570^{\mathrm{a}, \mathrm{A}}$ & $88.256 \pm 4.054^{\mathrm{a}, \mathrm{A}}$ \\
Colon & $58.053 \pm 5.044^{\mathrm{b}, \mathrm{B}}$ & $71.554 \pm 0.543^{\mathrm{a}, \mathrm{A}}$ & $72.804 \pm 3.971^{\mathrm{a}, \mathrm{A}}$ & $69.577 \pm 8.757^{\mathrm{a}, \mathrm{A}}$ \\
Feces & $18.427 \pm 1.522^{\mathrm{c}, \mathrm{B}}$ & $24.458 \pm 3.947^{\mathrm{b}, \mathrm{B}}$ & $35.198 \pm 3.270^{\mathrm{b}, \mathrm{A}}$ & $35.186 \pm 4.478^{\mathrm{b}, \mathrm{A}}$ \\
\hline \multicolumn{2}{c}{${ }^{\mathrm{a}, \mathrm{b}}$ Means in the same column followed by different lowercase letters are significantly different $(P<0.05)}$. \\
A,B Means in the same row followed by different uppercase letters are significantly different $(P<0.05$ ). \\
${ }^{1}$ Results are expressed as mean \pm standard error of means, $\mathrm{n}=6$. \\
${ }^{2}$ Treatments: Control = no L. casei ASCC 292, fructooligosaccharide (FOS), or maltodextrin; LF $=0.9 \%$ \\
(wt/wt) L. casei ASCC 292 and 2.5\% (wt/wt) FOS; LM = 0.9\% (wt/wt) L. casei ASCC 292 and 3.3\% (wt/wt) \\
maltodextrin; LFM = 0.9\% (wt/wt) L. casei ASCC 292, 2.5\% (wt/wt) FOS, and 3.3\% (wt/wt) maltodextrin.
\end{tabular}




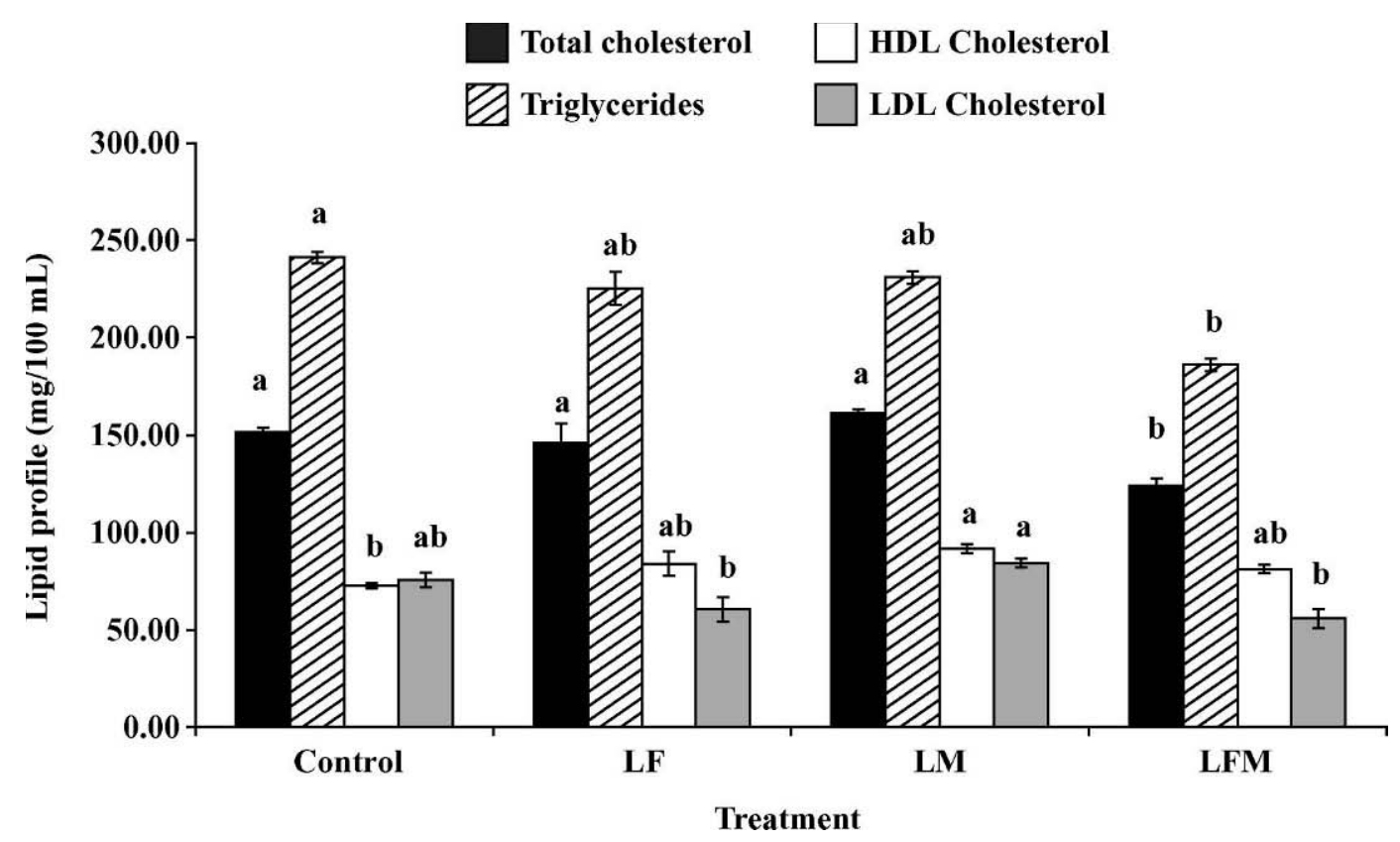

Figure 1. Total cholesterol, triglycerides, high-density lipoprotein cholesterol, and low-density lipoprotein cholesterol levels of rats fed 1 of 4 diets: Control = no L. casei ASCC 292, fructooligosaccharide (FOS), or maltodextrin; LF = 0.85\% (wt/wt) L. casei ASCC 292 and $2.40 \%$ (wt/wt) FOS; LM = 0.85\% (wt/wt) L. casei ASCC 292 and 3.40\% (wt/wt) maltodextrin; LFM = 0.85\% (wt/wt) L. casei ASCC 292, 2.40\% (wt/ wt) FOS, and $3.40 \%$ (wt/wt) maltodextrin. Results are expressed as mean \pm standard error of means, $\mathrm{n}=6$. ${ }^{\mathrm{a}, \mathrm{b}}$ Means within the same series followed by different lowercase letters are significantly different $(P<0.05)$.

Table 6. Population of total aerobes, staphylococci, Escherichia coli, and total coliforms from cecal, colon, and fecal contents of rats fed control and synbiotic diets ${ }^{1}$

\begin{tabular}{|c|c|c|c|c|}
\hline & \multicolumn{4}{|c|}{ Treatment $^{2}$} \\
\hline & Control & $\mathrm{LF}$ & LM & LFM \\
\hline & & (cfu/g of & sample) & \\
\hline \multicolumn{5}{|c|}{ Total aerobes } \\
\hline Cecum & $9.393 \pm 0.047^{\mathrm{a}, \mathrm{A}}$ & $9.929 \pm 0.014^{\mathrm{a}, \mathrm{A}}$ & $9.225 \pm 0.068^{\mathrm{a}, \mathrm{A}}$ & $9.229 \pm 0.083^{\mathrm{a}, \mathrm{A}}$ \\
\hline Colon & $9.413 \pm 0.032^{\mathrm{a}, \mathrm{A}}$ & $9.763 \pm 0.094^{\mathrm{a}, \mathrm{A}}$ & $9.032 \pm 0.011^{\mathrm{ab}, \mathrm{AB}}$ & $8.548 \pm 0.063^{\mathrm{a}, \mathrm{B}}$ \\
\hline Feces & $7.672 \pm 0.060^{\mathrm{b}, \mathrm{AB}}$ & $7.841 \pm 0.054^{\mathrm{b}, \mathrm{AB}}$ & $8.383 \pm 0.056^{\mathrm{b}, \mathrm{A}}$ & $7.156 \pm 0.053^{\mathrm{b}, \mathrm{B}}$ \\
\hline \multicolumn{5}{|c|}{ Staphylococci } \\
\hline Cecum & $7.502 \pm 0.031^{\mathrm{a}, \mathrm{B}}$ & $8.860 \pm 0.045^{\mathrm{a}, \mathrm{A}}$ & $9.149 \pm 0.089^{\mathrm{a}, \mathrm{A}}$ & $6.747 \pm 0.015^{\mathrm{a}, \mathrm{B}}$ \\
\hline Colon & $7.580 \pm 0.037^{\mathrm{a}, \mathrm{B}}$ & $8.484 \pm 0.074^{\mathrm{a}, \mathrm{A}}$ & $7.038 \pm 0.058^{\mathrm{b}, \mathrm{BC}}$ & $6.581 \pm 0.054^{\mathrm{a}, \mathrm{C}}$ \\
\hline Feces & $7.280 \pm 0.027^{\mathrm{a}, \mathrm{A}}$ & $7.658 \pm 0.180^{\mathrm{b}, \mathrm{A}}$ & $6.184 \pm 0.045^{\mathrm{c}, \mathrm{B}}$ & $6.257 \pm 0.064^{\mathrm{a}, \mathrm{B}}$ \\
\hline \multicolumn{5}{|c|}{ Escherichia coli } \\
\hline Cecum & $9.010 \pm 0.047^{\mathrm{a}, \mathrm{A}}$ & $8.448 \pm 0.064^{\mathrm{a}, \mathrm{AB}}$ & $8.540 \pm 0.021^{\mathrm{a}, \mathrm{AB}}$ & $7.948 \pm 0.013^{\mathrm{a}, \mathrm{B}}$ \\
\hline Colon & $9.027 \pm 0.062^{\mathrm{a}, \mathrm{A}}$ & $8.385 \pm 0.047^{\mathrm{ab}, \mathrm{AB}}$ & $8.330 \pm 0.034^{\mathrm{ab}, \mathrm{AB}}$ & $7.721 \pm 0.086^{\mathrm{ab}, \mathrm{B}}$ \\
\hline Feces & $7.415 \pm 0.062^{\mathrm{b}, \mathrm{A}}$ & $6.709 \pm 0.043^{\mathrm{b}, \mathrm{B}}$ & $7.688 \pm 0.082^{\mathrm{b}, \mathrm{A}}$ & $7.182 \pm 0.045^{\mathrm{b}, \mathrm{AB}}$ \\
\hline \multicolumn{5}{|c|}{ Coliforms } \\
\hline Cecum & $9.057 \pm 0.113^{\mathrm{a}, \mathrm{A}}$ & $9.215 \pm 0.017^{\mathrm{a}, \mathrm{A}}$ & $8.946 \pm 0.015^{\mathrm{a}, \mathrm{A}}$ & $8.054 \pm 0.111^{\mathrm{a}, \mathrm{B}}$ \\
\hline Colon & $9.050 \pm 0.108^{\mathrm{a}, \mathrm{A}}$ & $8.989 \pm 0.086^{\mathrm{a}, \mathrm{A}}$ & $8.688 \pm 0.071^{\mathrm{a}, \mathrm{A}}$ & $7.812 \pm 0.034^{\mathrm{a}, \mathrm{B}}$ \\
\hline Feces & $7.431 \pm 0.033^{\mathrm{b}, \mathrm{AB}}$ & $6.757 \pm 0.085^{\mathrm{b}, \mathrm{B}}$ & $7.641 \pm 0.054^{\mathrm{b}, \mathrm{A}}$ & $6.875 \pm 0.053^{\mathrm{b}, \mathrm{B}}$ \\
\hline
\end{tabular}

${ }^{\mathrm{a}-\mathrm{c}}$ Means in the same column followed by different lowercase letters are significantly different $(P<0.05)$.

${ }^{\mathrm{A}-\mathrm{C}}$ Means in the same row followed by different uppercase letters are significantly different $(P<0.05)$.

${ }^{1}$ Results are expressed as mean \pm standard error of means, $\mathrm{n}=6$.

${ }^{2}$ Treatments: Control $=$ no L. casei ASCC 292, fructooligosaccharide $(\mathrm{FOS})$, or maltodextrin; $\mathrm{LF}=0.9 \%$ (wt/wt) L. casei ASCC 292 and 2.5\% (wt/wt) FOS; LM = 0.9\% (wt/wt) L. casei ASCC 292 and 3.3\% (wt/wt) maltodextrin; LFM = 0.9\% (wt/wt) L. casei ASCC 292, 2.5\% (wt/wt) FOS, and 3.3\% (wt/wt) maltodextrin. 
Table 7. Population of total anaerobes, clostridia, bacteroides, and lactobacilli from cecal, colon and fecal contents of rats fed control and synbiotic diets ${ }^{1}$

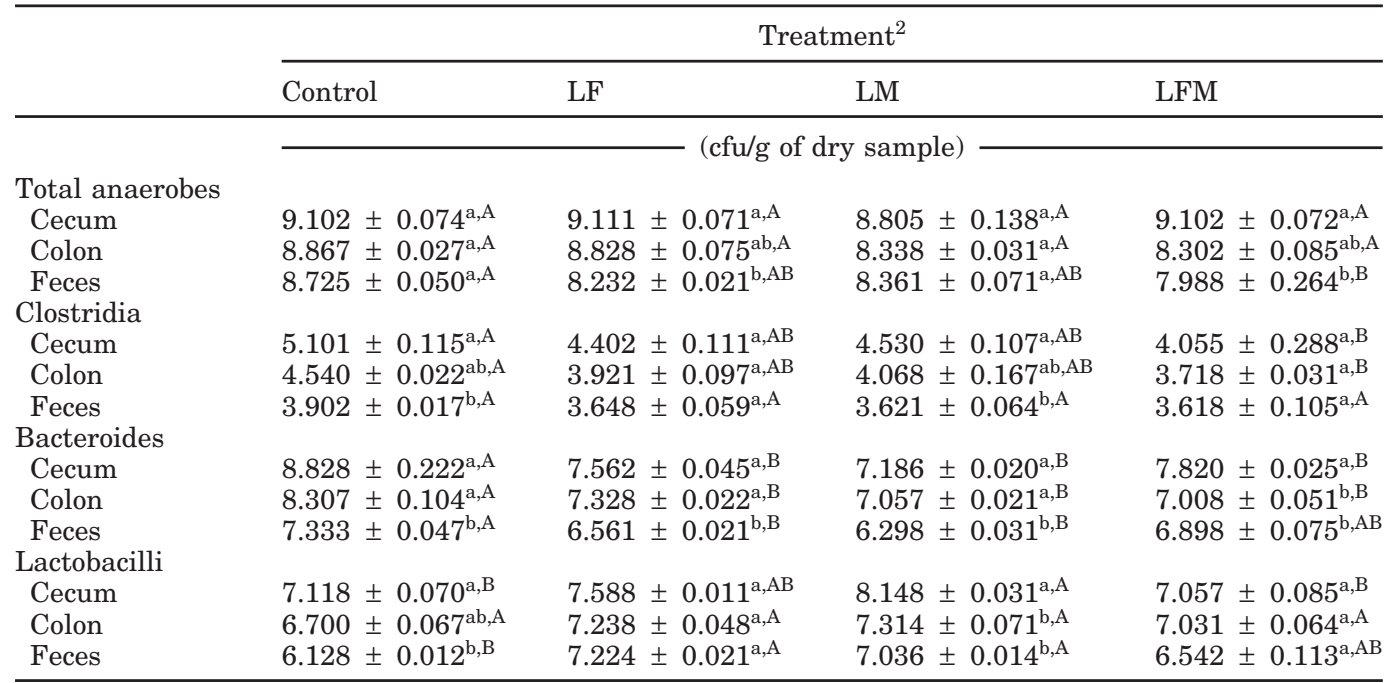

${ }^{\mathrm{a}, \mathrm{b}}$ Means in the same column followed by different lowercase letters are significantly different $(P<0.05)$.

${ }^{\mathrm{A}, \mathrm{B}}$ Means in the same row followed by different uppercase letters are significantly different $(P<0.05)$.

${ }^{1}$ Results are expressed as mean \pm standard error of means, $\mathrm{n}=6$.

${ }^{2}$ Treatments: Control = no L. casei ASCC 292, fructooligosaccharide $($ FOS $)$, or maltodextrin; $\mathrm{LF}=0.9 \%$ (wt/wt) L. casei ASCC 292 and 2.5\% (wt/wt) FOS; LM = 0.9\% (wt/wt) L. casei ASCC 292 and 3.3\% (wt/wt) maltodextrin; LFM = 0.9\% (wt/wt) L. casei ASCC 292, 2.5\% (wt/wt) FOS, and 3.3\% (wt/wt) maltodextrin.

\section{Concentration of Organic Acids}

The concentration of organic acids in different intestinal sites of rats is shown in Table 8. The concentration of acetic acid was consistent in the cecal and colon regions in rats fed the LF and LM diets, whereas a decrease in concentration after the region was seen in the control and LFM diets. Rats supplemented with the LM and LFM diets also showed a lower concentration of acetic acid in most intestinal contents compared with the control. All diets showed a gradient decrease in the concentration of butyric acid from samples obtained from cecum to feces. Rats supplemented with the LFM diet showed a significantly lower concentration of butyric acid compared with the control in all intestinal sites studied. Similar to the control diet, a gradient decrease in the concentration of formic acid was observed across intestinal regions from rats fed all synbiotic diets. The LF diet contributed to a higher concentration of formic acid in the cecal region compared with the control, whereas the LFM diet caused a decrease in the concentration of formic acid in most intestinal sites compared with the control. Concentration of propionic acid was least affected by all synbiotic diets, with insignificant changes across most intestinal gradients compared with that of the control. Only rats fed the LFM diet had significantly ( $P$ $<0.05$ ) higher amount of propionic acid in the cecum compared with the control and other treatment groups. The concentration of lactic acid was higher in the cecal region of rats fed the LM and LFM diets, and in the cecal and colonic regions of rats on the LF diet, compared with the control. However, the LM diet contributed to a drastic decrease in the concentration of lactic acid across all intestinal gradients, with the lowest amount detected in the fecal samples compared with the other diets.

\section{DISCUSSION}

This feeding trial was conducted to investigate the combined effect of $L$. casei ASCC 292 with FOS, L. casei ASCC 292 with maltodextrin, and $L$. casei ASCC 292 with FOS and maltodextrin on serum cholesterol, intestinal microflora, concentration of organic acids, and the translocation of lactobacilli using rats as a model. Body mass gain was in tandem with feed intake for all treatment diets, indicating a similar feed efficiency across all diets. Our results showed that the LM and LFM diets contributed to higher fecal moisture compared with the control diet. A major factor in determining the water content of the lumen is the osmolality of the contents; osmolality would be increased substantially by the presence of the unfermented carbohydrate used in this study. This is the basis for the use of nondigestible sugar alcohols and oligosaccharides as inexpensive bowel regulators and treatments for constipation in humans. Our results here indicated that the LM and LFM diets had higher laxative potential compared with the control. 
Table 8. Concentrations organic acids from cecal, colon and fecal content of rats fed control and synbiotic $\operatorname{diets}^{1}$

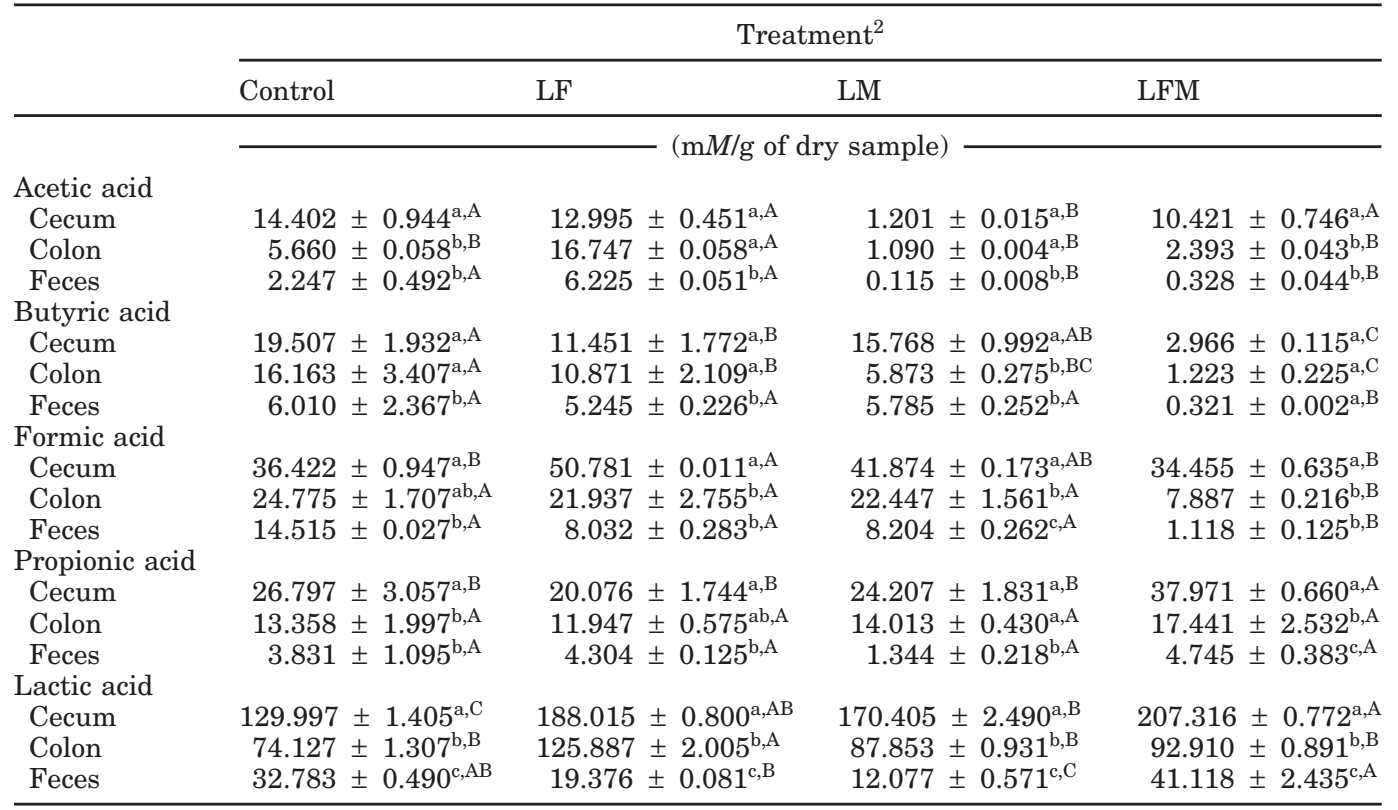

${ }^{a-c}$ Means in the same column followed by different lowercase letters are significantly different $(P<0.05)$.

${ }^{\mathrm{A}-\mathrm{C}}$ Means in the same row followed by different uppercase letters are significantly different $(P<0.05)$.

${ }^{1}$ Results are expressed as mean \pm standard error of means, $\mathrm{n}=6$.

${ }^{2}$ Treatments: Control $=$ no L. casei ASCC 292, fructooligosaccharide $(\mathrm{FOS})$, or maltodextrin; $\mathrm{LF}=0.9 \%$ (wt/wt) L. casei ASCC 292 and 2.5\% (wt/wt) FOS; LM = 0.9\% (wt/wt) L. casei ASCC 292 and 3.3\% (wt/wt) maltodextrin; LFM = 0.9\% (wt/wt) L. casei ASCC 292, 2.5\% (wt/wt) FOS, and 3.3\% (wt/wt) maltodextrin.

None of the synbiotic diets exhibited harmful translocation of lactobacilli to organs such as spleen, liver, and kidney. Results from this study clearly indicated that lactobacilli from all synbiotic diets were safe and did not translocate to other internal organs.

Overall, the combination of $L$. casei ASCC 292, fructooligosaccharide, and maltodextrin (LFM diet) produced lower total cholesterol and triglyceride levels. This supports our previous study on in vitro optimization, which reported optimum removal of cholesterol when $L$. casei ASCC 292 was used in the presence of FOS and maltodextrin (Liong and Shah, 2005c). The combination of $L$. casei ASCC 292 and maltodextrin (LM diet) beneficially increased the level of HDL cholesterol, whereas none of the synbiotics used altered the level of LDL cholesterol compared with the control. The hypocholesterolemic effect of the LFM diet may be due to various factors. A possible mechanism involves the alteration of lipid metabolism by short-chain fatty acids. Propionate was reported to inhibit fatty acid synthesis in vitro, and acetate is a lipogenic substrate (Delzenne and Kok, 2001). It was also found that hepatocytes isolated from rats fed an oligofructose diet had a $40 \%$ lower capacity to synthesize triacylglycerol from $\left[{ }^{14} \mathrm{C}\right]$ acetate than did control rats (Kok et al., 1996). In our study, results showed that the concentration of propionic acid in the ceca of rats fed
LFM was higher than the control, whereas the concentration of acetic acid in the fecal samples was comparatively lower. Results from our study indicated that the microbial fermentation of FOS and maltodextrin in the LFM diet induced the concentration of propionic acid that may have altered the cholesterol synthesis pathways, or decreased the concentration of acetic acid that may lead to a decreased lipogenesis.

Rats that were supplemented with diets containing FOS (LF and LFM) showed a constant lactobacilli count across cecal, colonic, and fecal samples, whereas the control group showed a decline in lactobacilli counts after the colonic region. Our previous in vitro study (Liong and Shah, 2005c) found that FOS was a good specific carbon source in modulating growth rate of $L$. casei ASCC 292, and thus may have exerted similar effects in this trial. In addition, the LFM diets had reduced total populations of clostridia. It was previously reported that an increase in bifidobacteria was often accompanied by a decrease in concentration of clostridia (Gibson et al., 1995). This study shows that a constant population of lactobacilli may have a similar effect. Although the FOS diets maintained a consistent lactobacilli count along the intestinal gradients, only the LM diet contributed to increased population of lactobacilli compared with the control. It was previously reported that malto- 
dextrin-like oligosaccharide had a lower rate of fermentation than FOS and was more fermentable in the distal part of the large intestine (Flickinger et al., 2000). Diet LFM also contributed to a decreased count of staphylococci and total coliforms in most intestinal samples, whereas the control diet exhibited a comparatively higher count. However, the LF diet increased the population of staphylococci compared with the control. This is supported by a previous study that reported better use of chicory oligofructose by staphylococci compared with synthetic oligofructose or even glucose (Roberfroid et al., 1998). The LFM diet also reduced population of $E$. coli in all intestinal samples compared with the control. Lactobacilli have been reported to inhibit the binding of enteropathogenic $E$. coli to intestinal cells (Bernet et al., 1994). Although most inhibition of pathogenic bacteria has been contributed by a decrease in intestinal $\mathrm{pH}$ (Swanson et al., 2002), the current study did not indicate such an association due to insignificant changes of $\mathrm{pH}$ along intestinal sites. In this study, these positive influences on pathogen populations may be supported by the higher concentration of lactic acid in cecum of rats fed the synbiotic diets compared with the control diet. Lactic acid is the major end product of lactate-producing lactobacilli (Swanson et al., 2002), and our results showed that the production of organic acids was dominated by lactic acid. Thus, the high production of lactic acid could indicate the possible antimicrobial capability on pathogenic microorganisms.

It must be noted that the concentration of acetic acid in rats supplemented fed the LM diet was lower compared with the other diets. A previous in vitro study (Liong and Shah, 2005d) reported that FOS was a better substrate for L. casei ASCC 292 for the production of acetic acid than was maltodextrin; this was confirmed in the current trial. Although the concentration of maltodextrin in the LM diet was higher than the concentration of FOS in the LF diet, the short-chain FOS used (average degree of polymerization of 4) would be more extensively fermented by the colonic bacteria than the longer chain maltodextrin (average degree of polymerization of 11), and thus contributing to higher concentration of acetic acid. It must also be noted that rats fed the LFM diet showed a much lower concentration of acetic acid in the lower bowel regions compared with the cecal region. We postulate that FOS in the LFM diet was quickly fermented by bacteria in the cecal region, resulting in a lower availability of FOS in the colonic regions thereafter; thus, contributing to a drastic decrease in acetic acid concentration after the cecal region. Results from this study also showed that a significantly lower concentration of butyric acid was detected in all intestinal sites studied from rats fed the LFM diet compared with control. It has been reported that short-chain fatty acids, especially butyrate, are the major intestinal fuel even in the presence of competing glucose (Windmueller and Spaeth, 1978). This led us to believe that the combination of FOS and maltodextrin may have synergistically accelerated the absorption of butyrate by colonocytes. Although the overall production of butyric acid may have been alternatively decreased, more work is needed to elucidate the absorption phenomenon, because if this postulation is true, the synergistic combination of $L$. casei ASCC 292, FOS, and maltodextrin could reduce the risk of colon cancer, which is often associated with decreased butyrate absorption in the bowel (Topping and Clifton, 2001).

Lactobacilli are lactate-producing bacteria, which could be observed from the higher concentration of lactic acid in cecal samples from rats fed the synbiotic diets compared with those fed the control. However, the concentration of lactic acid was higher in rats fed diets containing FOS than maltodextrin, which agrees with our previous in vitro study (Liong and Shah, 2005d). In that study, we reported that FOS was a better substrate for L. casei ASCC 292 to produce lactic acid compared with maltodextrin. In general, acetate is produced predominantly, followed by propionate (Roberfroid et al., 1998). Results from this study did not indicate such orientation. As reported in our previous study (Liong and Shah, 2005d), acetate was inhibited by the end-product of maltodextrin fermentation. We postulate that the fermentation of maltodextrin in the cecum and colon may have contributed to such end-product inhibition. In addition, we previously reported that FOS encouraged the production of propionic acid, which may have generated the higher concentration of propionic acid in this study.

\section{CONCLUSIONS}

The rats fed the synbiotic diets (LM and LFM) had higher moisture content in the feces compared with rats fed the control diet, indicating better laxative effects of the synbiotic diets. Diet LFM decreased the serum total cholesterol and triglyceride contents, whereas diet LM increased serum HDL cholesterol level. Diet LFM played a major role in decreasing populations of staphylococci, $E$. coli, coliforms, bacteroides, and clostridia. The increased population of lactobacilli contributed to a decreased population of clostridia and increased concentration of lactic acid. Diets containing FOS contributed to increased concentration of acetic acid. A much lower concentration of butyric acid was detected in the intestinal sites of rats fed the LFM diet compared with other diets, suggesting that the LFM diet accelerated butyrate absorption by colonocytes. This study showed that the combination of $L$. casei ASCC 292, FOS, and maltodextrin is beneficial for pathological cholesterol levels and 
healthier bowel microbial population without exhibiting harmful lactobacilli translocation.

\section{REFERENCES}

Argenzio, R. A., and D. J. Meuten. 1991. Short-chain fatty acids induce reversible injury of porcine colon. Dig. Dis. Sci. 36:1459-1468.

Bernet, M. F., D. Brassart, J. R. Neesar, and A. L. Servin. 1994 Lactobacillus acidophilus LA 1 binds to cultured human intestinal cell lines and inhibits cell attachment and cell invasion by enterovirulent bacteria. Gut 35:483-489.

Buddington, K. K., J. B. Donahoo, and R. K. Buddington. 2002. Dietary oligofructose and inulin protect mice from enteric and systemic pathogens and tumor inducers. J. Nutr. 132:472-477.

Chen, W. J. L., J. W. Anderson, and D. Jennings. 1984. Propionate may mediate the hypocholesterolemic effects of certain soluble plant fibres in cholesterol-fed rats. Proc. Soc. Exp. Biol. Med. 175:215-218.

Cotton, L. N., and C. H. White. 1992. Listeria monocytogenes, Yersinia enterocolitica, and Salmonella in dairy plant environments. J. Dairy Sci. 75:51-57.

Delzenne, N. M., and N. Kok. 2001. Effects of fructans-type prebiotics on lipid metabolism. Am. J. Clin. Nutr. 73:456S-458S.

Dubey, U. K., and V. V. Mistry. 1996. Effect of bifidogenic factors on growth characteristics of bifidobacteria in infant formulas. J. Dairy Sci. 79:1156-1163.

Flickinger, E. A. B., W. Wolf, K. A. Garleb, J. Chow, G. J. Leyer, P. W. Johns, and G. C. Fahey. 2000. Glucose-based oligosaccharides exhibit different in vitro fermentation patterns and affect in vivo apparent nutrient digestibility and microbial populations in dogs. J. Nutr. 130:1267-1273.

Gasser, F. 1994. Safety of lactic acid bacteria and their occurrence in human clinical infections. Bull. Inst. Pasteur 92:45-46.

Gibson, G. R., E. R. Beatty, X. Wang, and J. H. Cummings. 1995. Selective stimulation of bifidobacteria in the human colon by oligofructose and inulin. Gastroenterology 108:975-982.

Gibson, G. R., and M. B. Roberfroid. 1995. Dietary modulation of the human colonic microbiota: Introducing the concept of prebiotics. J. Nutr. 125:1401-1412.

Grunewald, K. K. 1982. Serum cholesterol levels in rats fed skim milk fermented by Lactobacillus acidophilus. J. Food Sci. 47:2078-2079.

Grunewald, K. K., and K. Mitchell. 1983. Serum cholesterol levels in mice fed fermented and unfermented acidophilus milk. J. Food Prot. 46:315-318.

Ishibashi, N., and S. Yamazaki. 2001. Probiotics and safety. Am. J. Clin. Nutr. 73:462S-470S.

Isolauri, E., P. V. Kirjavainen, and S. Salminen. 2002. Probiotics: A role in the treatment of intestinal infection and inflammation. Gut 50:54-59.

Jenkins, D. J., D. Cuff, T. M. Wolever, D. Knowland, L. Thompson, L. Cohen, and E. Prokipchuk. 1987. Digestibility of carbohydrate in an ileostomate: Relationship to dietary fibre, in vitro digestibility, and glycemic response. Am. J. Gastroenterol. 82:709-717.
Knowles, J. R., S. Roller, D. B. Murray, and A. S. Naidu. 2005. Antimicrobial action of carvacrol at different stages of dual-species biofilm development by Staphylococcus aureus and Salmonella enterica serovar Typhimurium. Appl. Environ. Microbiol. 71:797-803.

Kok, N., M. Roberfroid, and N. Delzenne. 1996. Involvement of lipogenesis in the lower VLDL secretion induced by oligofructose in rats. Br. J. Nutr. 76:881-890.

Liong, M. T., and N. P. Shah. 2005a. Acid and bile tolerance and cholesterol removal ability of lactobacilli strains. J. Dairy Sci. 88:55-66.

Liong, M. T., and N. P. Shah. 2005b. Bile salt deconjugation ability, bile salt hydrolase activity and cholesterol co-precipitation ability of lactobacilli strains. Int. Dairy J. 15:391-398.

Liong, M. T., and N. P. Shah. 2005c. Optimization of cholesterol removal by probiotics in presence of prebiotics using response surface methodology. Appl. Environ. Microbiol. 71:1745-1753.

Liong, M. T., and N. P. Shah. 2005d. Optimization of growth of Lactobacillus casei ASCC 292 and production of organic acids in the presence of fructooligosaccharide and maltodextrin. J. Food Sci. 70:M113-M120.

McBain, A. J., R. G. Bartolo, C. E. Catrenich, D. Charbonneau, R. G. Ledder, and P. Gilbert. 2003. Effects of triclosan-containing rinse on the dynamics and antimicrobial susceptibility of in vitro plague ecosystems. Antimicrob. Agents Chemother. 47:3531-3538.

Remesy, C., M. A. Levrat, L. Gamet, and C. Demigne. 1993. Cecal fermentations in rats on oligosaccharides (inulin) are modulated by dietary calcium level. Am. J. Physiol. Gastrointest. Liver Physiol. 264:G855-G862.

Roberfroid, M. B., J. A. E. Van Loo, and G. R. Gibson. 1998. The bifidogenic nature of chicory inulin and its hydrolysis products. $\mathrm{J}$ Nutr. 128:11-19.

Swanson, K. S., C. M. Grieshop, E. A. Flickinger, L. L. Bauer, B. W. Wolf, J. M. Chow, K. A. Garleb, J. A. Williams, and G. C. Fahey, Jr. 2002. Fructooligosaccharides and Lactobacillus acidophilus modify gut microbial populations, total tract nutrient digestibilities and fecal protein catabolite concentrations in healthy adult dogs. J. Nutr. 132:3721-3731.

Tannock, G. W., K. Munro, H. J. M. Harmsen, G. W. Welling, J. Smart, and P. K. Gopal. 2000. Analysis of the fecal microflora of human subjects consuming a probiotic product containing Lactobacillus rhamnosus DR20. Appl. Environ. Microbiol. 66:2578-2588.

Thompson, L. U., D. J. A. Jenkins, M. A. Amer, R. Reichert, A. Jenkins, and J. Kamulsky. 1982. Effect of fermented and unfermented milks on serum cholesterol. Am. J. Clin. Nutr. 36:1106-1111.

Topping, D. L., and P. M. Clifton. 2001. Short-chain fatty acids and human colonic functions: Roles of resistant starch and nonstarch polysaccharides. Physiol. Rev. 81:1031-1063.

Usman, and A. Hosono. 2000. Effect of administration of Lactobacillus gasseri on serum lipids and fecal steroids in hypercholesterolemic rats. J. Dairy Sci. 83:1705-1711.

Windmueller, H. G., and A. E. Spaeth. 1978. Identification of ketone bodies and glutamine as the major respiratory fuels in vivo for postabsorptive rat small intestine. J. Biol. Chem. 253:69-76.

Wise, M. G., and G. R. Siragusa. 2005. Quantitative detection of Clostridium perfringens in the broiler fowl gastrointestinal tract by real-time PCR. Appl. Environ. Microbiol. 71:3911-3916. 